Int. J. Dev. Biol. 50: 151-155 (2006)

doi: $10.1387 / \mathrm{ijdb} .052040 \mathrm{gb}$

\title{
Do lamellipodia have the mechanical capacity to drive convergent extension?
}

\author{
G. WAYNE BRODLAND* \\ Department of Civil Engineering, University of Waterloo, Waterloo, Canada
}

\begin{abstract}
Convergent extension (CE), a kinematic motif associated with several important morphogenetic movements in embryos, entails narrowing of a tissue in one in-plane direction and elongation in the other. Although the cell elongation and intercalation which accompany this process have been investigated and relevant genes and biochemical pathways have been studied in multiple organisms, a fundamental question that has not yet been answered is "Do the lamellipodia thought to drive these motions actually have the mechanical capacity to do so?" Here, we address this and a number of related issues using a state-of-the-art computational model which can replicate cell motions, changes in cell shape and tissue deformations. The model is based on the cell-level finite element approach of Chen and Brodland, but has additional features which allow it to model lamellipodium formation and contraction. In studying $C E$, computational models provide an important complement to molecular approaches because they reveal the "mechanical pathways" through which gene products must ultimately act in order to produce physical movements. The model shows that lamellipodia can drive CE, that they do so through cell intercalations and that the elongated cells characteristic of $C E$ arise only when adjacent tissues resist convergence, a result which we confirm experimentally.
\end{abstract}

KEY WORDS: embryo mechanics, morphogenetic movement, computational modeling, $\gamma-\mu$ model

\section{Introduction}

The morphogenetic movements by which embryos are formed are among the most striking motions observed in nature. These motions are initiated, driven and regulated through a complex interplay of genes, gene products and mechanical forces (Wallingford et al., 2002; Keller, 2004; Ninomiya et al., 2004). During certain morphogenetic movements, including gastrulation and neurulation, sheets of tissue converge in one in-plane direction and extend in the other, a process called convergent extension (CE) (Mittenthal and Jacobson, 1990; Keller et al., 2000). At the onset of $C E$, cellular protrusions that previously arose with random orientations begin to be polarized in the mediolateral direction (Wallingford et al., 2002). Signaling pathways that involve planar cell polarity (PCP) (Wallingford and Harland, 2004), Wnt/Ca2+ and other molecules (Heisenberg et al., 2000; Wallingford et al., 2000; Wallingford et al., 2002; Lu et al., 2004) serve to initiate, direct and stabilize these protrusions, although the details are still under investigation.

These protrusions, called lamellipodia, take the form of thin, planar projections that grow out from a cell $A$ (Fig. 1A) by actin polymerisation (Shafer, 2004), push their way (Williams-Massen et al., 1998) between an adjacent pair of cells B and $C$ and may ultimately contact the next nearest neighbor (Bailly and Condeelis, 2002) D. When contact is made, the lamellipodium may contract and in doing so pull (Bailly and Condeelis, 2002) on cell D. It is widely held (Keller, 2000; Elul and Keller, 2000; Wallingford et al., 2002; Keller, 2004) that the action of these lamellipodia cause the cells to intercalate with each other in the mediolateral direction and thus drive contraction in the mediolateral direction and extension in the cephalocaudal direction.

This sequence of events can be considered a "mechanical pathway" and has important parallels with the molecular pathways familiar to developmental biologists. The mechanical pathway we hypothesize drives CE starts with the formation and contraction of oriented lamellipodia. Lamellipodium action then causes cells to intercalate with each other and finally, intercalation gives rise to deformation and to tissue reshaping. The events in this pathway involve subcellular components, cells and tissues and they span length scales ranging from microns to millimeters. While this hypothesized sequence of events may be true, its

Abbreviations used in this paper: CE, convergent extension; FEM, finite element method. 
A
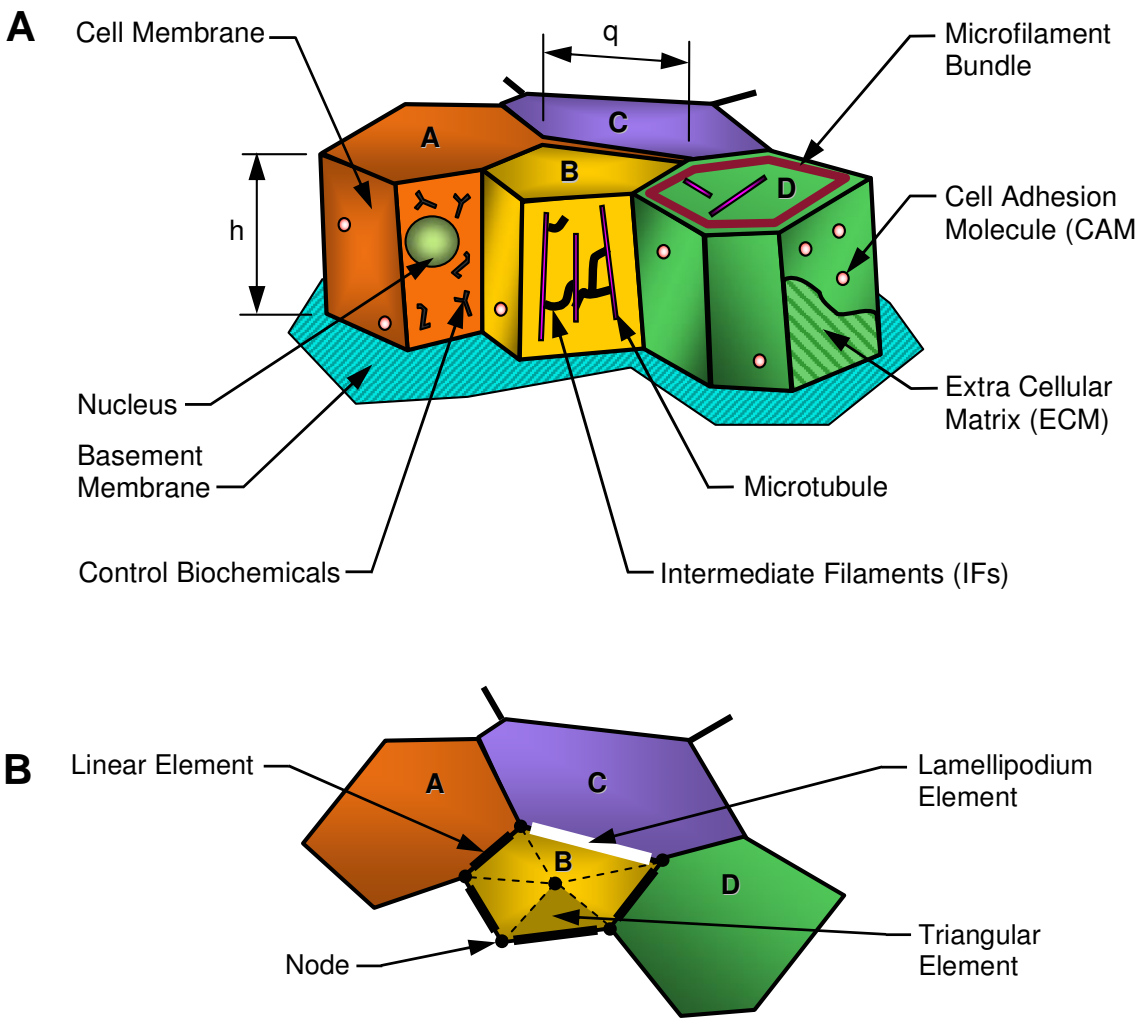

Fig. 1. A section of a monolayer epithelium. (A) Shown schematically, are the cell nucleus and gene products, cytoskeletal components and the extracellular matrix (ECM) and basement membrane. A lamellipodium is shown extending from cell $A$ between its neighbors $B$ and $C$ and contacting the next neighboring cell $D$. The area of the new interface formed is $q$. The lamellipodium may form entirely from cell $A$ or in part from $A$ and in part from $D$. Connections at the lamellipodium ends are assumed to be durable enough to withstand any subsequent lamellipodium contraction. (B) In the corresponding finite element model, the cytoplasm, networks of filaments and basement membrane are represented by triangular elements and are assumed to have viscosity $\mu$. The mechanical effects of the microfilaments, cell membrane, cell adhesion molecules and ECM are represented by rod-like elements along the interfaces and are assumed to generate a net interfacial tension $\gamma$. These elements are herein referred to as "linear" elements because they have the form of a line segment. It should be noted, however, that they do not have linear mechanical properties.

mechanical plausibility has not previously been investigated. Testing is crucial since the mechanics of cell-cell interactions and morphogenetic movements can be highly counterintuitive and the outcomes of mechanical interactions can be completely unexpected (Clausi and Brodland, 1993; Brodland and Clausi, 1995; Davidson et al., 1999; Brodland, 2004; Hardin and Walston, 2004). Indeed, "Without [such testing], we are only engaging in handwaving and wishful thinking about the performance of our models" (Gordon, 1985).

Just as there are established procedures for testing hypotheses about molecular pathways, so there are for testing hypotheses about mechanical ones. These include computational models based on the finite element method (Brodland and Chen, 2000; Brodland, 2004; Zienkiewicz et al., 2005), a method that has been used extensively in engineering and physics for several decades and that is now finding a key role in biology and various other model types, some of which have previously been used to model lamellipodia (Palsson, 2001) and to investigate CE (Zajac et al., 2003). Computational models are important in biology because they can reveal how the individual components of a complex system interact with each other to produce a specific result (Doyle, 2001; Endy and Brent, 2001). Unlike man-made (i.e., "engineered") systems, which are typically built up by successively refining a basic system, often by increasing its complexity, biological systems generally present in a complex form and computational models are essential for deconstructing them (Endy and Brent, 2001; Kitano, 2002). Another important feature of a computational model is that, unlike an experiment, it allows one to consider a system that contains only components specified by the user, making possible definitive statements regarding the capacity of specific structures to drive particular actions (Clausi and Brodland, 1993; Taber et al., 1995; Davidson et al., 1999; Chen and Brodland, 2000; Brodland and Chen, 2000; Palsson, 2001; Brodland, 2002; Brodland and Veldhuis, 2002; Brodland, 2003; Brodland, 2004; Brodland and Wiebe, 2004).

Philosophically, we assume that gene expression gives rise to specific biochemical pathways and that these pathways ultimately are responsible for construction and regulation of a variety of subcellular structural components (Fig. 1A). Thus, biochemical pathways can interface to the beginning of mechanical ones, such as the one outlined above for CE. In a monolayer epithelium, the structural components that are built include the cell membrane and its associated structures, microfilaments, microtubules, intermediate filaments, cell adhesion molecules, extracellular matrix and basement membrane.

Like Chen and Brodland (2000), we assume that the cell membrane, its associated proteins, microfilaments and cell adhesion molecules collectively give rise to an equivalent interfacial tension $\gamma$ that acts along each cell-cell interface. The cell cytoplasm with its embedded filamentous networks and organelles are assumed to have viscosity $\mu$. The detailed calculations used in the determination of these mechanical properties have been reported elsewhere (Chen and Brodland, 2000; Brodland, 2002; Brodland, 2004).

Gamma-mu models that have been implemented numerically using the finite element method have been quite successful in predicting cell motions, cell reshaping and tissue deformation. They have also provided significant insights into how forces generated by sub-cellular structures give rise to mechanical effects at the cell and tissue levels (Chen and Brodland, 2000; Brodland and Chen, 2000; Brodland, 2002; Brodland and Veldhuis, 2002; Brodland, 2003; Brodland, 2004; Brodland and Wiebe, 2004). Here, we present a $\gamma-\mu$ finite element model which has been modified so that it can model lamellipodia. We use this model to investigate the mechanical capacity of the "mechanical pathways" which we hypothesize to drive CE. 


\section{The Computational Model}

In a finite element model, the object of interest is broken into elements that are sufficiently small and simple that each element can be described mathematically. The mathematical steps required to construct the equations for each such element and to assemble the element equations that result into a system of simultaneous equations representing the mechanical system as a whole are somewhat complicated (Brodland and Chen, 2000; Zienkiewicz etal., 2005). However, the main steps can be outlined so that the basis of the method can be understood, although this understanding is not crucial to grasping the conclusions drawn from the model.

Like most cell-based computational models (Brodland, 2004), the one used here assumes that energy is associated with each cell-cell interface. For an interface of height $h$ and length $q$, this energy is equal to $\gamma \mathrm{A}$ where $\mathrm{A}=\mathrm{qh}$ is the area of the interface and $\gamma$ is a constant of proportionality that can be interpreted as the interfacial tension along each cell-cell boundary, as it is here. To model this force, linear elements (Fig. 1B) are placed along each cell-cell interface and are assumed to carry a constant tension $\gamma \mathrm{h}$ (Chen and Brodland, 2000). If a lamellipodium extends and fills an interface between two cells $B$ and $C$ (Fig. 1A), the one original interface between the cells essentially becomes replaced by two and the linear element along that boundary is then made to carry a tension of $2 \gamma \mathrm{h}$.

To model the cell cytoplasm, including its organelles and embedded networks of filaments, each $n$-sided cell is broken into $\mathrm{n}$ triangular elements that completely cover the cell area (Fig. 1B) and that are assigned a viscosity $\mu$ (Chen and Brodland, 2000; Brodland, 2003). The amount of mechanical resistance provided by the cytoplasm depends on the epithelium thickness, an operational detail that is taken into account in the numerical implementation.

For each assumed time increment, $\Delta t$, during which the model runs, the vector sum of the interfacial tensions that impinge at each triple junction is calculated and written into a global force vector $f$. The viscous stiffness of each of the triangular elements representing the cytoplasm is then calculated and these are "summed" in a certain fashion (Chen and Brodland, 2000; Zienkiewicz etal., 2005) to produce a stiffness matrix Kdescribing the whole system. By solving the large system of equations, $K$ $\Delta u=f$, that results, one obtains a highly accurate approximation of the incremental displacements $\Delta u$ that would occur at each node during the time increment $\Delta t$. In essence, this mathematical procedure finds the unique set of nodal displacements (or velocities $\Delta u / \Delta t$ ) that would produce viscous forces in the cytoplasm that just balance the otherwise imbalanced interfacial tension forces. In the mechanics literature, this mechanism of deformation would be called creep flow.

As it runs, the model reveals the incremental displacements that assemblies of cells with specified initial geometries and mechanical properties would undergo, making no assumptions regarding the behavior that will result. A special algorithm is used to accommodate cell rearrangement (Chen and Brodland, 2000) and element contributions to fand $K$ are recalculated for each time increment based on the current geometry. The resulting process of "computational discovery" reveals how forces generated at the sub-cellular, cellular and tissue levels interact with each other and with any imposed boundary conditions to produce motions and changes of shape in cells and tissues.

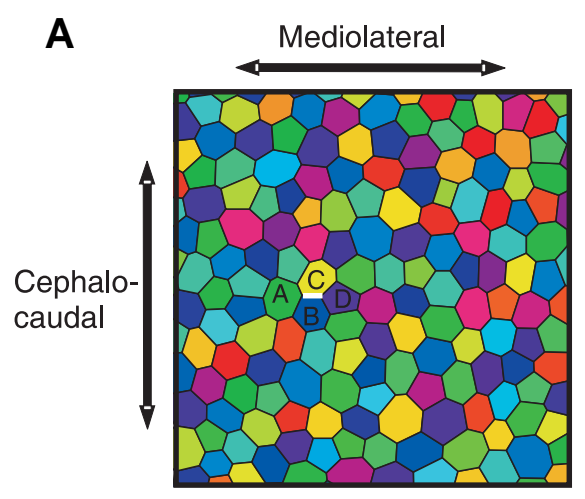

B
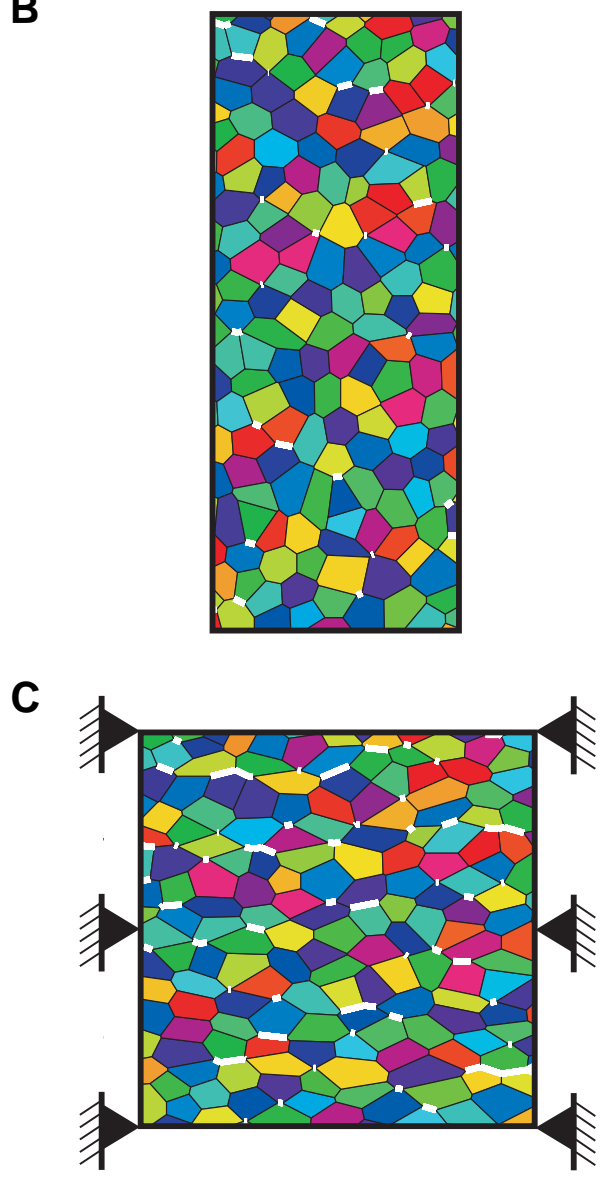

Fig. 2. Simulations in which mediolaterally-oriented lamellipodia form. (A) At each time step in the simulations, a specified number of cells, like the one labeled $A$, are chosen at random and a lamellipodium (labeled with a white bar) is assumed to form along whichever of its radial boundaries is most nearly aligned with the mediolateral direction. Cells have been colored to facilitate visual tracking between images. (B) If the lateral edges of the tissue are not restrained, the tissue undergoes convergent extension, but the cell texture remains essentially isotropic. (C) If the lateral edges of the tissue are restrained against inward motion, the cells elongate in the mediolateral direction. Figure parts $(B, C)$ correspond to a time when on average each cell has generated and contracted one lamellipodium. 


\section{Results and Discussion}

Figure 2 shows frames from simulations where lamellipodia with preferential mediolateral orientations are assumed to form in the mediolateral direction (the horizontal axis of the figure). While only a portion of the 400-cell model is shown in print in order that the shapes of individual cells can be seen easily, the full model, which consists of 2323 elements and 1202 nodes in its initial configuration, is shown in Supplementary Videos 1 and 2. There, the power of lamellipodia to drive CE in unrestrained tissues (Video 1) and to drive cell reshaping in restrained ones (Video 2) is illustrated vividly.

Other simulations show that when a single mediolaterally oriented lamellipodium pulls a pair of cells, A and D (Fig. 1A) together, neighboring cells are drawn in behind cells $A$ and $D$ and are pushed out of the way of cells $B$ and $C$, thereby producing a four leaf clovershaped pattern of displacements (Fig. 3A). When lamellipodia act successively at many different locations throughout the tissue, the cumulative effect, which might be considered a superposition of these local disturbances, results in a CE pattern (Fig. 3B) similar to that observed in real embryos (Burnside and Jacobson, 1968; Jacobson and Lofberg, 1969).

If cells $A$ and $D$ do not straddle the midsagittal plane, the more medial cell will displace laterally as they move together, explaining why experimentally observed cells do not move monotonically towards the medial axis (Ninomiya et al., 2004). Tension applied to cells $A$ and $D$ where the lamellipodium contacts them explains why pointed features (Miller et al., 1995) are observed there. The simulations also show that, like directed mitosis (Williams-Masson, 1998; Brodland and Veldhuis, 2002), lamellipodium action is quite efficient, changing the aspect ratio of a free, initially square region (Fig. 2A) to 2.5 (Fig. 2B) by the time that the cells have on average each produced and contracted one lamellipodium.

In Supplementary Video 1, from which Fig. 2B was taken, the
A

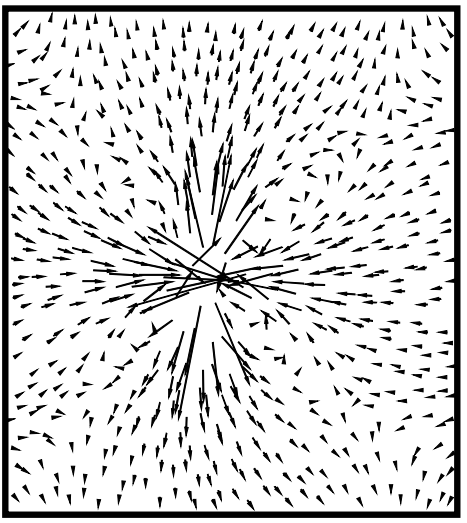

B

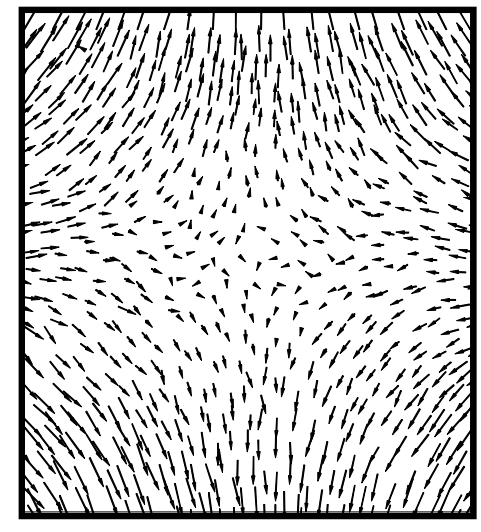

Fig. 3 Flow fields produced by lamellipodia. (A) The flow field produced by contraction of a single lamellipodium at the center of the region shown. The field of view corresponds approximately to that shown in Supplementary Video 1 and arrows show the directions and relative magnitudes of the displacements that occur during the time required for the one lamellipodium to contract and vanish. (B) The flow field produced by the action of many lamellipodia. Arrows indicate the magnified displacements of each node between the first and last frames of Supplementary Video 1. If the edges of the tissue are restrained, random looking displacements are produced (Supplementary Video 2). edges of the tissue are unrestrained and the average cell aspect ratio (Brodland and Veldhuis, 2002), $\kappa$, is 1.03. If the edges of the model tissue are restrained so that they cannot move, the cells stretch in the direction in which they are carrying tension (along the convergence axis) and their average aspect ratio is $\kappa=1.59$. Computer simulations and experiments show that when a tissue is stretched by external forces (Brodland and Wiebe, 2004) its cells stretch in the direction of tissue elongation. These and other simulations, experiments and analyses (Chen and Brodland, 2000; Brodland and Wiebe, 2004) show that cell elongation always correlates with the direction of the tension carried by the cells. Thus, curiously, when external forces dominate, cells elongate in the direction of tissue elongation while when lamellipodia dominate, they elongate normal to it.

To evaluate these numerical predictions, cell shape measurements (Iles et al., 2004) were made on epithelia from gastrulation stage amphibian (Ambystoma mexicanum) embryos where lamellipodia are highly active. Approximately 100 cells just cephalic to the blastopore along the midline of four intact stage 12.5 gastrulae were measured from high magnification digital images. In vivo, lateral restraint is provided by the adjacent tissues (Jacobson and Gordon, 1976), as in Fig. 2C and Supplementary Video 2 and cells stretch in the convergence direction until their average aspect ratios are $\kappa=1.65 \pm 0.2$ (indicated variation is from embryo to embryo), consistent with the predictions of the model. Cells in three corresponding explants, which like the simulations in Fig. 2B and Supplementary Video 1 were not laterally restrained, exhibited an aspect ratio of only $\kappa=1.05 \pm 0.05$. This ratio was consistent with model predictions for an unrestrained tissue and the difference between the experimental values for restrained and unrestrained tissues was significant at the level $P<0.001$. That the model can so accurately predict cell shapes, tissue deformations and interactions between the two of them in the presence of lamellipodia action suggests that its basic tenets are sound.

The model shows that CE can be understood to involve the following mechanical pathway:

1) Genes construct the system of structural components shown in Fig. 1A (this step can be viewed as a transition from a series of molecular pathways that build the individual components, to a mechanical one),

2) Under the control of specific gene products (another molecular input), lamellipodia are made to form in the mediolateral direction,

3) When the lamellipodia contract and pull pairs of cells (A and D in Fig. 1) together, localized displacements occur (Fig. 3A) and

4a) If the tissue is not restrained, these local displacements accumulate (Fig. 3B) to produce cell intercalation and a tissue-level response that is recognizable as CE.

$4 b)$ If the tissue is restrained, the local displacements give rise to cell elongation (Fig. $3 \mathrm{C}$ ).

4c) If the tissue is partially constrained, a combination of cell elongation and $\mathrm{CE}$ is possible (not shown).

The steps in this mechanical pathway involve scales ranging from the subcellular (Steps 1 and 2) to the cellular (Step 3) to the tissue level (Step 4). Without advanced computational models, like the one used here, it would be difficult to identify and decipher multiscale pathways of this kind. 


\section{Towards the future}

If current efforts continue, it is probable that the primary molecular pathways associated with CE will be largely identified within the next few years. Although that will be an important achievement, it cannot, by itself, provide a complete explanation of how CE occurs because it could not explain the mechanical forces present and how they interact to produce morphogenetic movements. Specifically, if mechanical forces suddenly ceased operating, then regardless of any molecules present, morphogenetic movements would also stop instantly. Similarly, although the computational model presented here showed that lamellipodia have the mechanical capacity to drive convergent extension, without the molecular literature it cannot explain how the subcellular structures through which it works arise. To obtain a comprehensive understanding of embryo morphogenesis, a strong partnership (Brent, 2004) is required between molecular biologists and mechanicians. Then, molecular pathways can be connected not just to each other, but to mechanical pathways like the one explored here. Cross-connections of this kind are crucial to the advancement of biomechanics and mechanobiology. Molecular biology and mechanics can be thought of as each holding some of the pieces needed to complete a jigsaw puzzle. Each puzzle piece represents an insight that can only be made from its distinctive perspective and to complete the picture, both must work together.

\section{Acknowledgements}

Funding was provided by the Canadian Institutes of Health Research (CIHR). Simulations were carried out by Jim Veldhuis and embryo data were collected by Jen Sweny and Richard Benko.

\section{References}

BAILLY, M. and CONDEELIS, J. (2002). Cell motility: insights from the backstage. Nat. Cell Biol. 4: E292-294.

BRENT, R. (2004). A partnership between biology and engineering. Nature Biotech. 22: $1211-1214$.

BRODLAND, G.W. and CLAUSI, D.A. (1995). Cytoskeletal mechanics of neurulation: Insights obtained from computer simulation. Biochem. and Cel/ Biol. 73: 545-553.

BRODLAND, G.W. and CHEN, H.H. (2000). The mechanics of heterotypic cell aggregates: Insights from computer simulations. ASME J. Biomech. Eng. 122: 402-407.

BRODLAND, G.W. (2002). The differential interfacial tension hypothesis (DITH): a comprehensive theory for the self-rearrangement of embryonic cells and tissues. J. Biomech. Eng. 124: 188-197.

BRODLAND, G.W. and VELDHUIS, J.H. (2002). Computer simulations of mitosis and interdependencies between mitosis orientation, cell shape and epithelia reshaping. J. Biomech. 35: 673-681.

BRODLAND, G.W. (2003). New information from cell aggregate compression tests and its implications for theories of cell sorting. Biorheology 40: 273-277.

BRODLAND, G.W. (2004). Computational modelling of cell sorting, tissue engulfment and related phenomena: A review. Appl. Mech. Rev. 57: 47-76.

BRODLAND, G.W. and WIEBE, C.J. (2004). Mechanical effects of cell anisotropy on epithelia. Comput. Methods Biomech. Biomed. Engin. 7 (2): 91-99.

BURNSIDE, M.B. and JACOBSON, A.G. (1968). Analysis of morphogenetic movements in the neural plate of the newt Taricha torosa. Dev. Biol. 18: 537-552.

CHEN, H.H. and BRODLAND, G.W. (2000). Cell-level finite element studies of viscous cells in planar aggregates. J. Biomech. Eng. 122: 394-401.

CLAUSI, D.A. and BRODLAND, G.W. (1993). Mechanical evaluation of theories of neurulation using computer simulations. Development 118: 1013-1023.
DAVIDSON, L.A., OSTER, G.F., KELLER, R.E. and KOEHL, M.A.R. (1999). Measurements of mechanical properties of the blastula wall reveal which hypothesized mechanisms of primary invagination are physically plausible in the sea urchin Strongylocentrotus purpuratus. Dev. Biol. 209: 221-238.

DOYLE, J. (2001). Beyond the spherical cow. Nature 411: 151-152.

ELUL, T. and KELLER, R. (2000). Monopolar protrusive activity: a new morphogenic cell behavior in the neural plate dependent on vertical interactions with the mesoderm in xenopus. Dev. Biol. 224: 3-19.

ENDY, D. and BRENT. R. (2001). Modelling cellular behaviour. Nature 409: 391-395.

GORDON, R. (1985). A review of the theories of vertebrate neurulation and their relationship to the mechanics of neural tube birth defects. J. Embryol. Exp. Morph. 89 Supplement: 229-255.

HARDIN, J. and WALSTON, T. (2004). Models of morphogenesis: The mechanisms and mechanics of cell rearrangement. Curr. Opin. Genet. Dev. 14: 399-406.

HEISENBERG, C. et al. (2000). Silberblick/Wnt11 mediates convergent extension movements during zebrafish gastrulation. Nature 405: 76-81.

ILES, P.J., CLAUSI, D.A. and BRODLAND, G.W. (2004). Estimation of average cell shape from digital images of cellular surfaces. IEEE Proceedings of the First Canadian Conference on Computer and Robot Vision. May 17-19, 2004:273-278.

JACOBSON, C.O. and LÖFBERG J. (1969). Mesoderm movements in the amphibian neurula. Zool. Bidrag, Uppsala 38: 233-239.

JACOBSON, A.G. and GORDON, R. (1976). Changes in the shape of developing vertebrate nervous system analyzed experimentally, mathematically and by computer simulation. J. Exp. Zool. 197: 191-246.

KELLER, R. et al. (2000). Mechanisms of convergence and extension by cell intercalation. Phil. Trans. R. Soc. Lond. B 355: 897-922.

KELLER, R. (2000). Shaping the vertebrate body plan by polarized embryonic cell movements. Science 298: 1950-1954.

KELLER, R. (2004). Developmental biology: Heading away from the rump. Nature 430: 305-306.

KITANO, H. (2002). Computation systems biology. Nature 420: 206-210.

$\mathrm{LU}, \mathrm{X}$. et al. (2004). PTK7/CCK-4 is a novel regulator of planar cell polarity in vertebrates. Nature 430: 93-98.

MILLER, J., FRASER, S.E. and MCCLAY, D. (1995). Dynamics of thin filopodia during sea urchin gastrulation. Development 121 (8): 2501-2511.

MITTENTHAL, J.E. and JACOBSON, A.G. (1990). The mechanics of morphogenesis in multicellular embryos. In Biomechanics of Active Movement and Deformation of Cells. (ed. N. Akkas). pp. 295-401. Berlin: Springer-Verlag.

NINOMIYA, H., ELINSON, R.P. and WINKLBAUER, R. (2004). Antero-posterior tissue polarity links mesoderm convergent extension to axial patterning. Nature 430: 364-367.

PALSSON, E. (2001). A three-dimensional model of cell movement in multicellular systems. Future Gen. Comput. Sys. 17: 835-852.

SHAFER, D.A. (2004). Cell biology: barbed ends rule. Nature 430: 734-5.

TABER, L.A., LIN, I.E. and CLARK, E.B. (1995). Mechanics of cardiac looping. Dev. Dyn. 203: 42-50.

WALLINGFORD, J.B. et al. (2000). Dishevelled controls cell polarity during Xenopus gastrulation. Nature 405: 81-85.

WALLINGFORD, J.B., FRASER, S.E. and HARLAND, R.M. (2002). Convergent extension: The molecular control of polarized cell movement during embryonic development. Dev. Cel/2 (6): 695-706.

WALLINGFORD, J.B. and HARLAND, R.M. (2004). Neural tube closure requires Dishevelled-dependent convergent extension of the midline. Development 129: 5815-5825.

WILLIAMS-MASSON, E.M., HEID, P.J., LAVIN, C.A. and HARDIN, J. (1998). The cellular mechanism of epithelial rearrangement during morphogenesis of the Caenorhabditis elegans dorsal hypodermis. Dev. Biol. 204: 263-276.

ZAJAC, M., JONES, G.L. and GLAZIER, J.A. (2003). Simulating convergent extension by way of anisotropic differential adhesion. J. Theor. Biol. 222:247259.

ZIENKIEWICZ, O.C., TAYLOR, R.L. and ZHU, J.Z. (2005). The Finite Element Method: Its Basis and Fundamentals. Butterworth-Heinemann, Woburn, MA. 